\title{
Triple-Negative Breast Cancer with Dermatomyositis: A Case Report and Literature Review
}

\author{
Xiao Chen, Aoxiang Chen, Chaoqi Liu, Bin Zhang \\ The First Department of Breast Cancer, Tianjin Medical University Cancer Institute and Hospital, National Clinical Research Center for Cancer, \\ Tianjin, People's Republic of China \\ Correspondence: Bin Zhang, The First Department of Breast Cancer, Tianjin Medical University Cancer Institute and Hospital, National Clinical \\ Research Center for Cancer, Huanhuxi Road, Hexi District, Tianjin, 300060, People’s Republic of China, Email eeflying@I63.com
}

\begin{abstract}
Purpose: Dermatomyositis (DM) is an autoimmune inflammatory myopathy, the onset of which is sometimes associated with some malignancies and sometimes appears as a symptom of paraneoplastic syndrome. The main symptoms include progressive proximal muscle weakness, extra muscular manifestations such as dyspnea or dysphagia, and skin changes. There is currently no standardized treatment for breast cancer associated with DM.

Patients and Methods: We report a 45-year-old woman with a palpable mass over the left external breast along with diffuse erythema on the shoulder, orbital edema, voice hoarseness, dyspnea, and weakness of the extremities. Needle aspiration biopsy and imaging suggested malignancy. Thus, breast-conserving surgery and pectoral myotomy biopsy+ skin excision biopsy were performed. Pathology confirmed triple-negative breast cancer (TNBC) and DM. Two days after surgery, the patient's orbital edema, voice hoarseness, dyspnea, and weakness of the extremities were significantly reduced, and the erythema subsided significantly. Left axillary lymph node dissection was performed after postoperative adjuvant chemotherapy, and the nodes were negative. The patient was recovering well, and follow-up showed no signs of recurrence or metastasis.

Conclusion: Be alert for the presence of malignancy when a breast cancer patient presents with DM, a test for dermatologists, rheumatologists, and oncologists. This case demonstrated the effectiveness of breast-conserving surgery combined with radiotherapy for early-stage triple-negative breast cancer with DM, even without further treatment for DM to reduce the symptoms. In conclusion, the treatment plan for these patients depends on the presentation of the tumor and DM.
\end{abstract}

Keywords: dermatomyositis, triple-negative breast cancer, paraneoplastic syndrome, breast-conserving surgery, case report

\section{Introduction}

Breast cancer is the most common malignancy in women, and the different subtypes of breast cancer exhibit considerable heterogeneity. ${ }^{1}$ Triple-negative breast cancer (TNBC) is a type of breast cancer that lack of expression estrogen receptor, progesterone receptor, and human epidermal growth factor receptor 2 (HER2) and accounts for approximately 10-24\% of all breast cancers. ${ }^{2}$ Compared with other subtypes, it is characterized by high aggressiveness, high recurrence rates, high metastatic potential, and poor prognosis. ${ }^{3-5}$ Neoadjuvant chemotherapy is currently the primary treatment for TNBC and significantly improves patient prognosis. ${ }^{6}$ Early diagnosis, individualized treatment, and regular review of TNBC are essential to prolong the life of patients and prevent recurrence.

$\mathrm{DM}$ is a rare autoimmune inflammatory myopathy that primarily involves the skin and muscles, with an incidence of approximately 9.63 per $1,000,000 .^{7}$ Skin lesions are usually the first symptoms and include erythematous rashes with or without orbital edema. Gottron's papules are often seen in bone joints. At the same time there are some other skin manifestations such as capillary dilation and shawl sign. Muscle involvement often presents as symmetrical proximal muscle weakness with or without muscle pain, and patients may also present with systemic manifestations including arthralgia, dyspnea, dysphagia, dysphonia, and vocal difficulties. ${ }^{8,9}$ According to epidemiological statistics, $15-30 \%$ of 
cases of DM are associated with malignancies. ${ }^{10}$ A study from Asia showed that DM is the second most common disease associated with breast cancer in women. ${ }^{11}$ There are no treatment guidelines related for breast cancer associated with $\mathrm{DM}$, and individualized treatment is based on the clinical cases. This study describes the case of one patient diagnosed with TNBC and DM and a treatment plan that achieved significant remission.

\section{Patients and Methods}

We report a case of a 45-year-old non-menopausal Asian woman with no family history of tumors and no history of drug abuse. She presented with a painless palpated mass in the left breast but did not seek medical care. One month later, diffuse erythema appeared on the upper body, including the anterior chest, dorsal shoulder, lateral upper limbs, abdomen, without other specific discomforts. A dermatologist at another hospital considered allergic dermatitis and gave oral antiallergy medication without significant relief. Five months later, the patient developed orbital edema, voice hoarseness, dyspnea, limb weakness, and the deepening of the rash color. The dermatologist considered tumor-associated dermatomyositis and recommended further examination of the left breast mass. The patient was admitted to our hospital. The patient found red-to-violet colored poikilodermatous patches on the anterior chest, back of the shoulder, and outside the upper arm, with lighter colored abdominal erythema and poorly defined borders (Figure 1). There was also facial edema, relatively severe around the eyes; the voice was altered from before, and hoarseness was present. There were also signs of muscle involvement: dyspnea and asymmetrical decrease in strength in proximal muscles (including cervical flexors) (score of 4/5), without changes in distal muscle (5/5). There was no purplish rash around the eyelids or orbits and no maculopapular rash on the metacarpophalangeal joints. Autoimmune-related indicators were negative. Aspartate transaminase and lactate dehydrogenase were elevated. Potassium, sodium, and calcium ions were decreased.

Mammography showed a firm, painless, fixed nodule of approximately $2 \mathrm{~cm}$ was palpable in the upper outer left breast and no definite mass was palpable in the contralateral breast or axilla. A Doppler ultrasound showed a left breast mass measuring approximately $1.5 \times 2.0 \times 1.4 \mathrm{~cm}$, which was considered breast cancer (BI-RADS: 4C) (Figure 2). Magnetic resonance imaging (MRI) showed 1) a mass in the supra-axillary area of the left breast (about $2.1 \times 1.9 \times$ $1.8 \mathrm{~cm}$ )-breast cancer (BI-RADS 5), and peri-tumoral edema; 2) multiple lymph nodes in the left axilla; and 3) diffuse heterogeneous signal in the pectoralis major, latissimus dorsi, and part of the scapular muscle (Figure 3). The pathological diagnosis of the breast needle aspiration biopsy was "invasive carcinoma of the breast." Tumor-

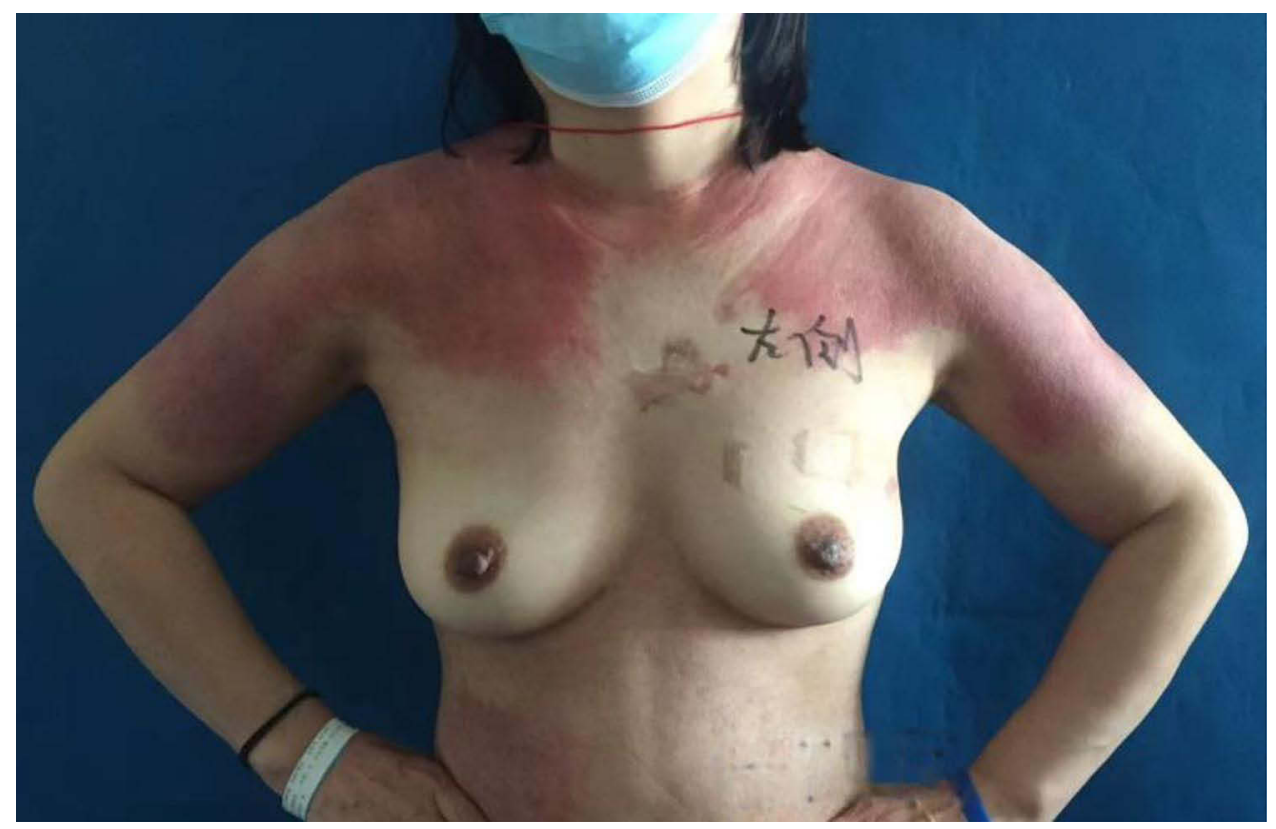

Figure I Rash: on admission. 


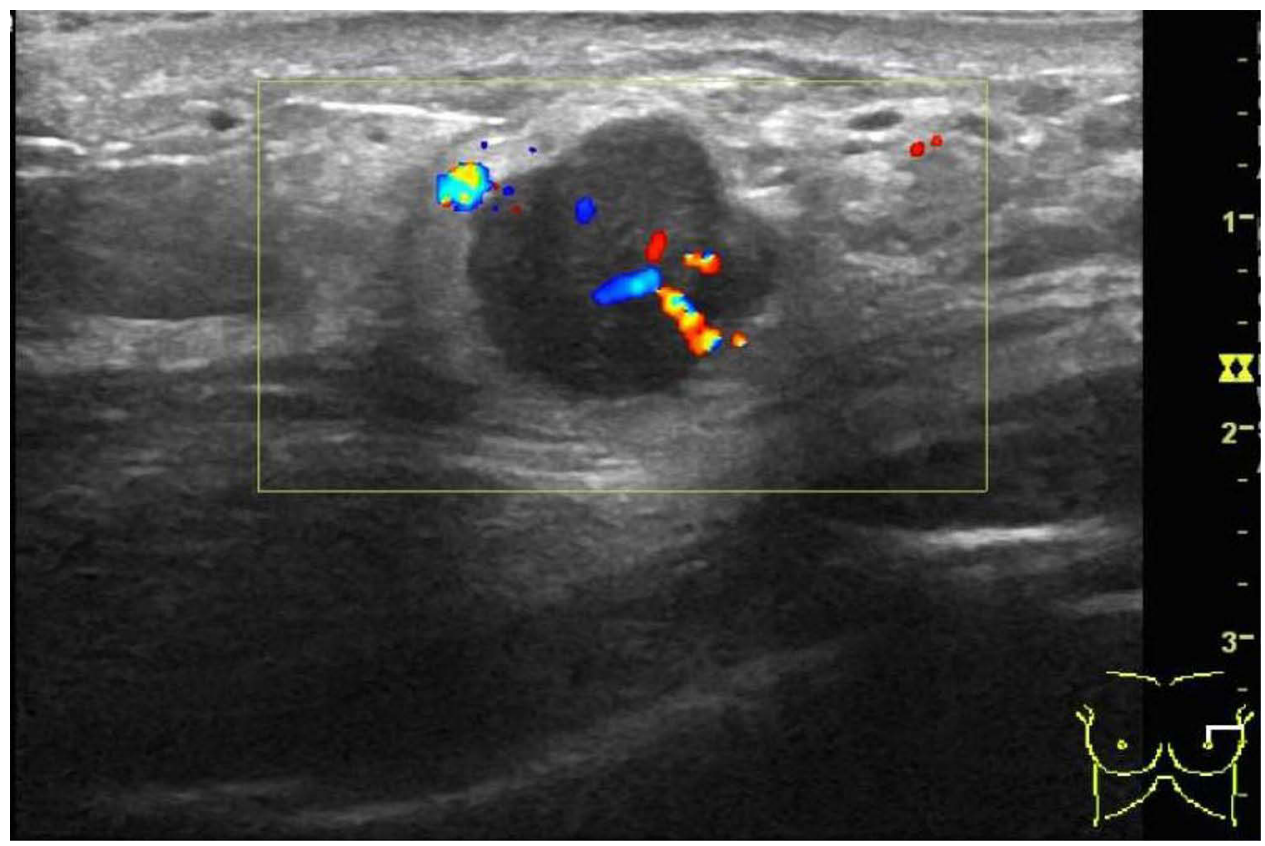

Figure 2 Breast ultrasound showed a $1.5 \times 2.0 \times 1.4 \mathrm{~cm}$ mass in the left breast.

associated DM was diagnosed by the oncologist and rheumatologist. To relieve the patient's current symptoms related to $\mathrm{DM}$ and at the same time, the patient insisted on breast-conserving treatment, left breast cancer breast-conserving surgery and skin and pectoral muscle excision biopsy were performed. Postoperative pathology confirmed that the left breast mass was invasive ductal carcinoma (Figure 4), and immunohistochemistry revealed that estrogen, progesterone receptor, and HER-2 expression was negative, suggesting TNBC. Skin and pectoral muscle biopsies suggested DM. Dyspnea, weakness, and hoarseness were significantly relieved 2 days after surgery. The edema was reduced. One week postoperatively, the rashes subsided significantly (Figure 5), and symptoms such as dyspnea, malaise, and hoarseness disappeared.

Postoperatively, the patient received two cycles of chemotherapy with $400 \mathrm{mg}$ of albumin paclitaxel (at a dose of $222 \mathrm{mg} / \mathrm{m} 2$ ). After the second cycle, the patient developed a generalized rash with localized blisters, which was considered drug allergy, and was given oral prednisone for symptom relief. The chemotherapy regimen was changed

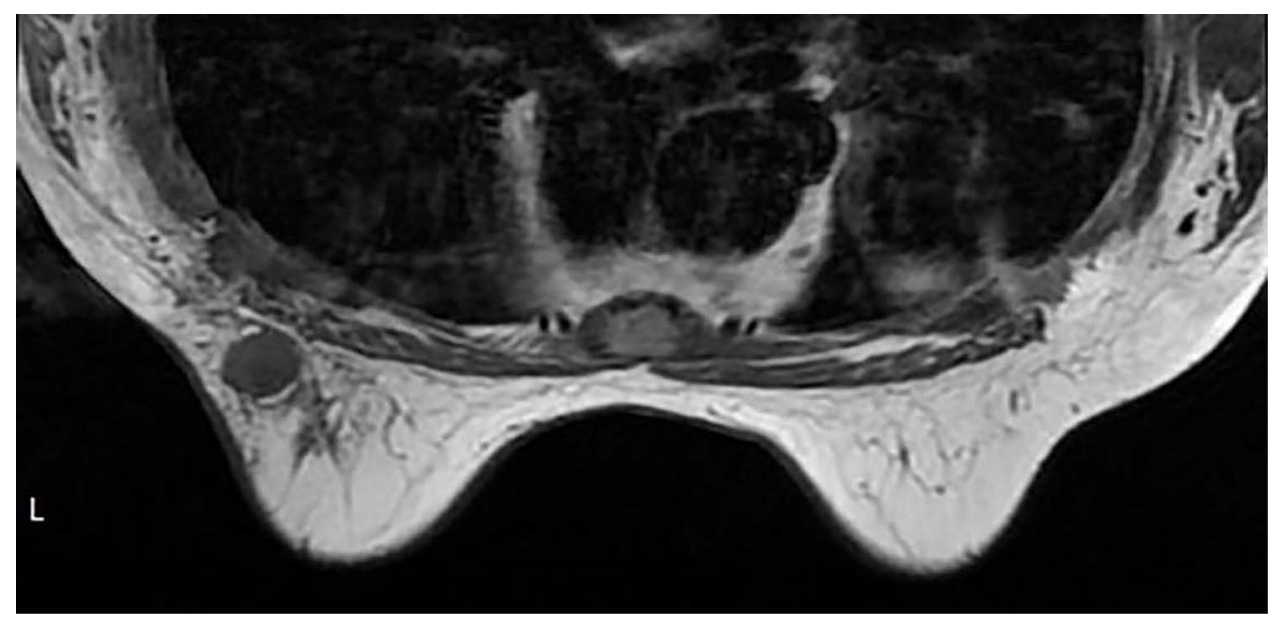

Figure 3 Breast MRI. 


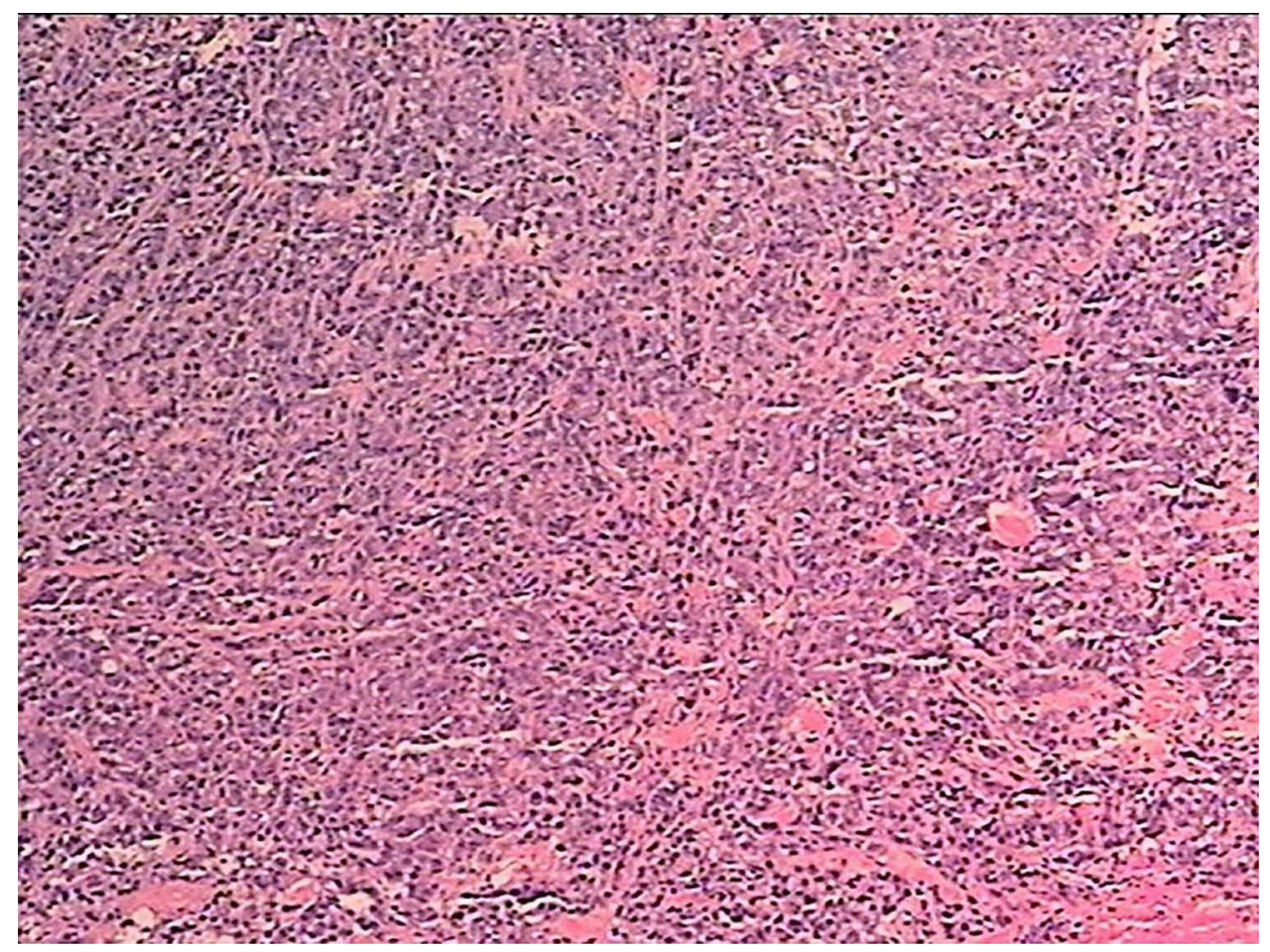

Figure 4 Histological examination shows invasive ductal carcinoma $(\mathrm{H}-\mathrm{E}$, original magnification, $\times 100)$.

to two cycles of $900 \mathrm{mg}$ carboplatin to relieve stage IV myelosuppression, and the carboplatin dose was changed to $700 \mathrm{mg}$ from the fifth- to the eighth cycle. Postoperative breast ultrasound and PET/CT did not exclude left axillary lymph node metastasis (Figure 6), and the patient underwent left axillary lymph node dissection. Lymph nodes were

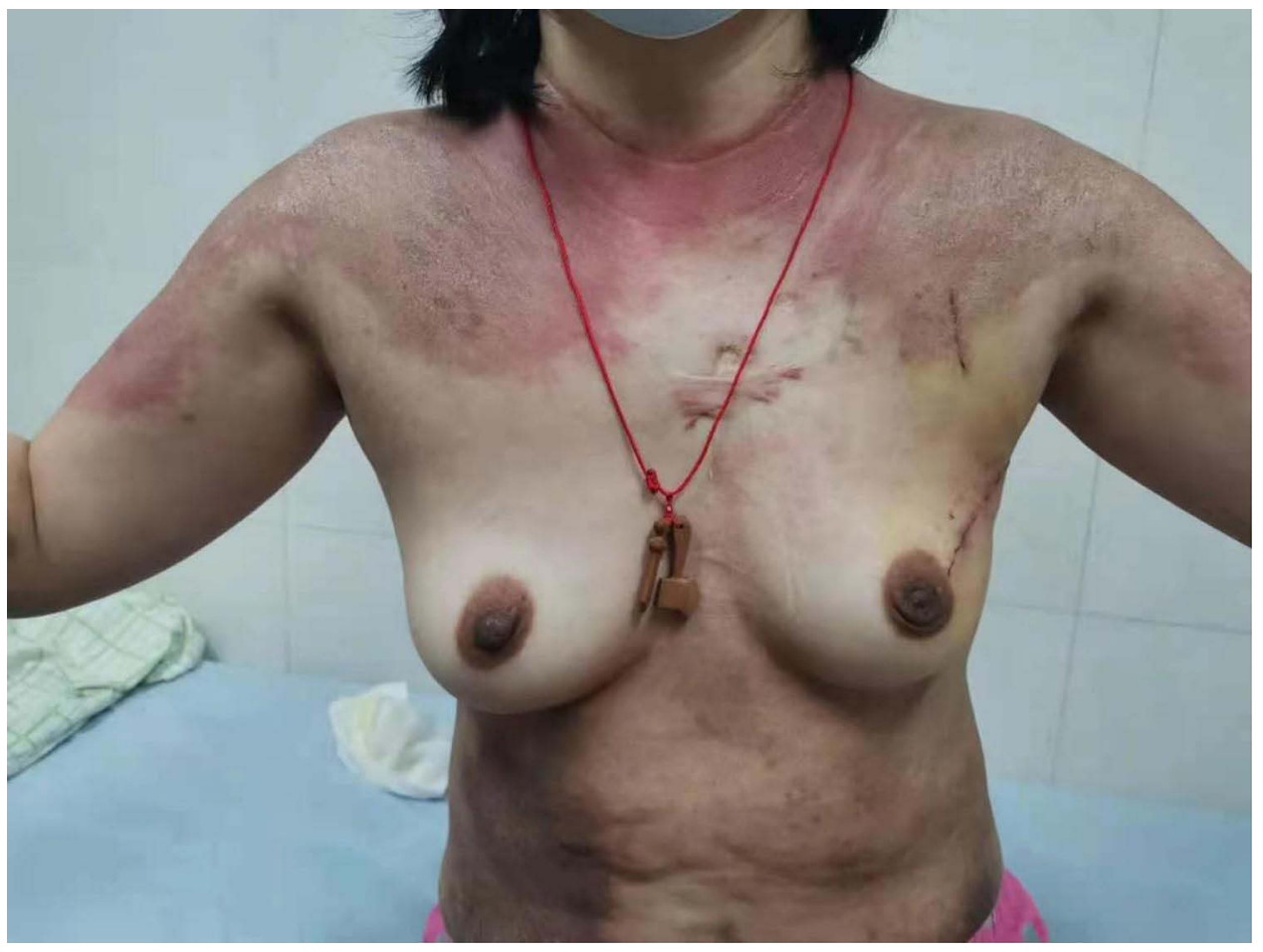

Figure 5 I week after breast cancer surgery. 


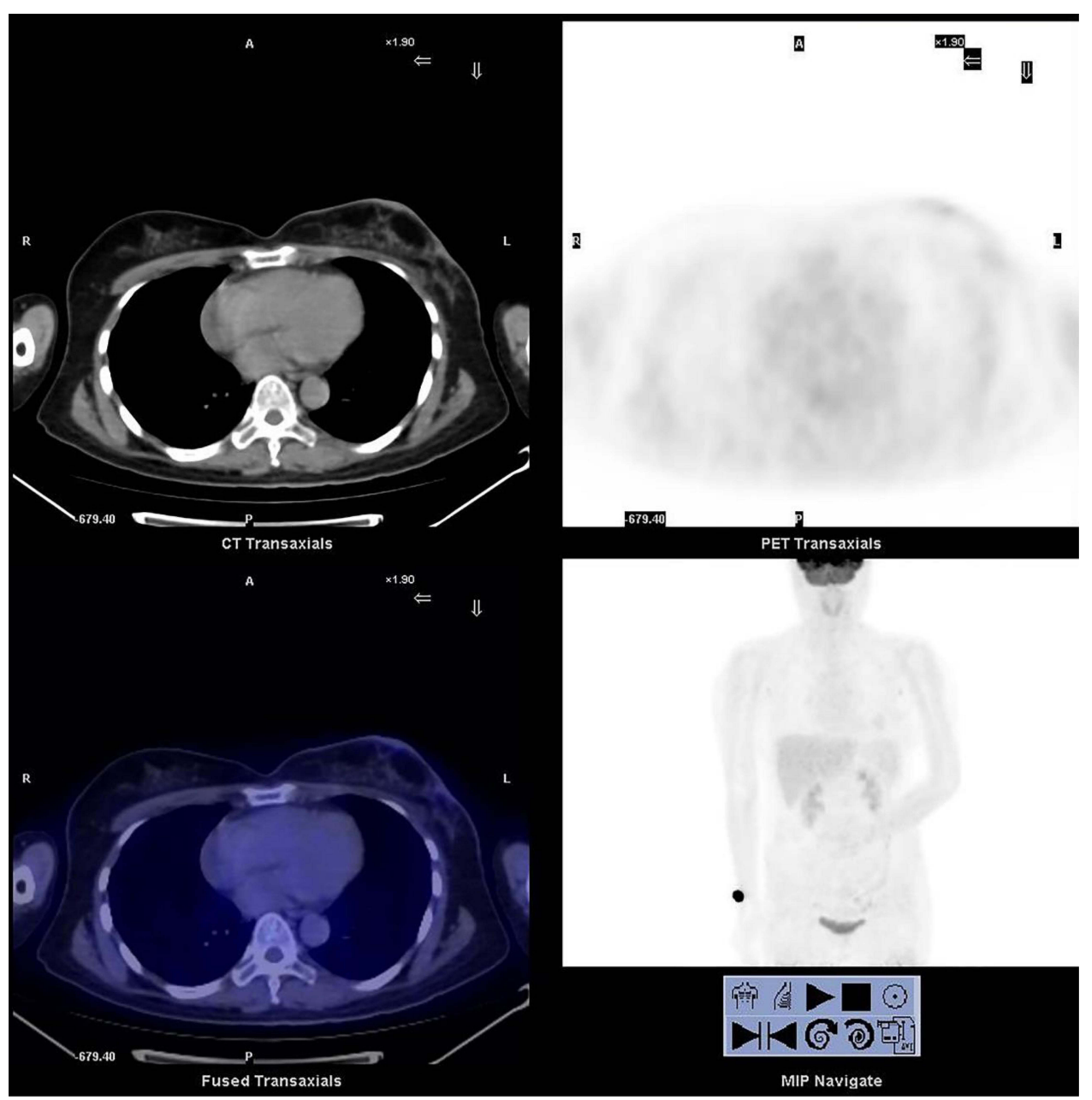

Figure 6 Postoperative PET/CT of the breast.

histopathologically negative, and the patient subsequently received adjuvant radiotherapy. The patient received a pathologic complete response. Computed tomography showed no metastasis in the chest, abdomen, and pelvis, and bone scan revealed no bone metastasis. At 1 year postoperatively, the abdominal erythema subsided, and erythema on the shoulder was significantly reduced (Figure 7).

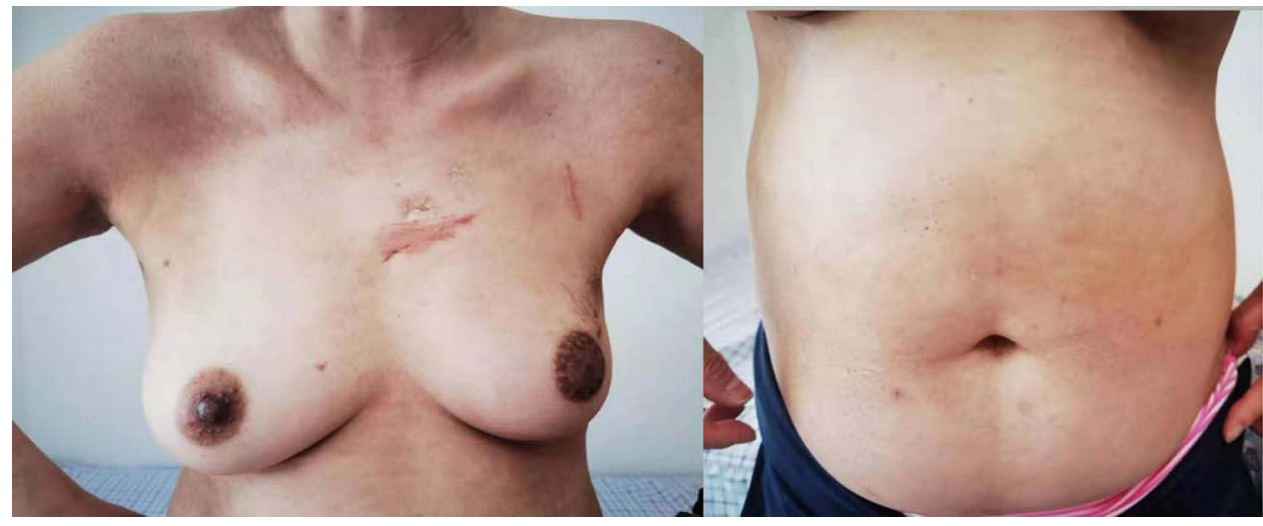

Figure 7 I year after breast cancer surgery. 
The 1.5-year follow-up showed that our patient had no evidence of local recurrence or distant disease, and effective resolution of DM symptoms. Our patient is currently taking no medications.

\section{Discussion}

Symptoms of DM mainly involve the skin and muscles. Consider tumor recurrence when symptoms of the skin reappear after treatment. ${ }^{12}$ The long-term use of some chemotherapeutic agents such as capecitabine can lead to the appearance of DM-like symptoms, which need to be carefully distinguished from the hand-foot syndrome. ${ }^{13}$ A meta-analysis of 28 cohort studies showed that age $>45$ years, male gender, cutaneous vasculitis, skin necrosis, dysphagia, rapid onset of myositis (4 months from initial symptoms to diagnosis), and elevated CK, ESR, and PCR increased the risk of developing tumors in patients with DM. ${ }^{14}$ Our patient presented four of these risk factors.

Epidemiological studies have described and confirmed the current link between breast cancer and DM. People with DM are six times more likely to develop malignancies than the general population. However, the exact mechanism of occurrence is still unclear. ${ }^{15}$ Myositis-Specific Antibodies (MSAs) are closely related to the diagnosis, treatment and prognosis of DM, mainly anti-Mi2 antibodies, anti-MDA5 antibodies, anti-NXP2 antibodies, anti-TIF1 antibodies, antiJo-1 antibodies, anti-SAE, etc. ${ }^{16}$ Tumor cells express high levels of MSAs, such as anti-TIF1 antibodies, which are clearly associated with potentially malignant tumors. It is hypothesized that anti-TIF1 antibodies are produced in antitumor immune responses. ${ }^{17}$ At the same time, Casciola-Rosen et al found that the antigenic fingerprints in some adenocarcinomas, such as breast cancer, are highly similar to the regenerating muscle cells of myositis patients, speculating that the antitumor response will initiate the immune response of DM. ${ }^{18}$ On the other hand, damaged and regenerated muscle tissue in DM patients is a major source of MSAs. Anti-NXP2 antibodies are associated with a higher risk of malignancy. ${ }^{19}$ The anti-Jo-1 antibody is also one of the risk factors for tumor development in patients. ${ }^{20}$ Therefore, when a tumor complicated with DM occurs, excision of the tumor lesion is a more effective treatment for relieving the symptoms of DM..$^{21,22}$

Neoadjuvant chemotherapy is the primary systemic treatment for TNBC, which improves pathologic complete remission, increasing surgical success, reducing the extent of surgery, and allowing assessment of treatment response. ${ }^{23}$ The treatment of DM is mainly based on systemic corticosteroids. ${ }^{24}$ There is no standardized treatment for breast cancer associated with DM, and individualized treatments need to be based on the patient's condition. It is important to note that the timing of treatment for DM and breast cancer is also crucial. Dias et al reported that severe muscle symptoms should be controlled with steroids before radical excision of breast cancer. However, treatment is still required due to poor DM remission. ${ }^{25}$ In another patient, mastectomy and postoperative adjuvant therapy were then started after remission of rash symptoms; the patient recovered better from DM after surgery, but the associated rash symptoms occasionally recurred. ${ }^{26}$ After Dr. Piras et al gave both neoadjuvant and DM-related treatment, the patient's DM symptoms had largely recovered, mastectomy was performed, and the patient recovered well. ${ }^{10}$ Controlling DMrelated symptoms before surgery reduces postoperative wound healing from DM, but the overall time to control the disease is longer than patients treated surgically first.

Some clinicians believe it is critical to address tumor-related issues first, as DM may manifest as a paraneoplastic syndrome. Inaguma et al initiated oral prednisolone therapy and neoadjuvant chemotherapy concurrently and treat with total mastectomy after significant tumor shrinkage, but still require hormone therapy for DM postoperatively. ${ }^{27}$ Similarly Hendren et al opted for surgery after significant tumor shrinkage with neoadjuvant chemotherapy and postoperative antiDM treatment with Steroid drugs and methotrexate, but rashes and muscle weakness were poorly controlled. ${ }^{12}$ For HER2-positive patients, DM symptoms improved little when treatment was directed at DM alone, and when adjuvant therapy with chemoimmunotherapy was switched, DM subsided quickly. ${ }^{28}$ Like $\mathrm{Hu}$ et al ${ }^{15}$ and Zhang et al, ${ }^{29}$ in the present case, we chose to remove the primary tumor lesion by lumpectomy first. The patient's postoperative musclerelated manifestations were significantly relieved, the rash subsided significantly, which further confirmed that DM is a manifestation of the paraneoplastic syndrome in the present case.

When patients have relatively severe muscular symptoms, the primary tumor lesion can be removed first as mentioned. $^{21,22}$ In some patients, further treatments of DM are even not necessary. Suppose the patient simply has severe cutaneous manifestations rash symptoms of $\mathrm{BC}$ combined with DM without muscle symptoms, the cutaneous 
manifestations can be relieved by neoadjuvant therapy combined with or without DM-related treatment before surgery to promote wound healing and prevent postoperative infection. For the choice of surgical approach, when combined with DM, most surgeons choose total mastectomy. If the patient's tumor is eligible for breast conservation and the patient has the will to conserve breast, dermatomyositis should not be a stumbling block for breast-conserving surgery. ${ }^{30}$ One of the limitations of this study is the short follow-up (latest 18 months postoperative), and we will continue to follow the patient's review results and changes.

\section{Conclusion}

We offer a patient with triple-negative breast cancer combined with paraneoplastic dermatomyositis that significantly regressed dermatomyositis symptoms with breast conservation and adjuvant therapy. Thus, an adult patient is diagnosed with DM, clinicians should consider the importance of early cancer screening, effective oncological treatments based on the presentation of tumor and DM, improved prognosis, and the need for multidisciplinary physician collaboration in the management of malignancy and dermatomyositis.

\section{Abbreviations}

DM, dermatomyositis; TNBC, triple-negative breast cancer; MRI, magnetic resonance imaging; PET-CT, positron emission tomography-computerized tomography; MSAs, myositis-specific antibodies.

\section{Consent for Publication}

This case study was approved by the Ethics Committee of Tianjin Medical University Cancer Institute and Hospital. Written informed consent was obtained from the patient for publication of this case report and any accompanying images.

\section{Acknowledgments}

The authors would like to thank the patient and her family who trusted us with the care, and all of the doctors and staff who helped in this study.

\section{Disclosure}

The authors declare no conflicts of interest in this work.

\section{References}

1. Siegel RL, Miller KD, Fuchs HE, Jemal A. Cancer statistics, 2021. CA Cancer J Clin. 2021;71(1):7-33. doi:10.3322/caac.21654

2. Vaidyanathan K. The challenge of triple negative breast cancers. Indian J Clin Biochem. 2014;29(3):267-268. doi:10.1007/s12291-014-0453-1

3. Haffty BG, Yang Q, Reiss M, et al. Locoregional relapse and distant metastasis in conservatively managed triple negative early-stage breast cancer. $J$ Clin Oncol. 2006;24(36):5652-5657. doi:10.1200/JCO.2006.06.5664

4. Dent R, Trudeau M, Pritchard KI, et al. Triple-negative breast cancer: clinical features and patterns of recurrence. Clin Cancer Res. 2007;13(15 Pt 1):4429-4434. doi:10.1158/1078-0432.CCR-06-3045

5. Calderon LE, Black CA, Rollins JD, et al. Synthesis of radiolabeled technetium- and rhenium-luteinizing hormone-releasing hormone ((99m)Tc/ Re-Acdien-LHRH) conjugates for targeted detection of breast cancer cells overexpressing the LHRH receptor. ACS Omega. 2021;6(3):1846-1856. doi:10.1021/acsomega.0c03991

6. Yin L, Duan JJ, Bian XW, Yu SC. Triple-negative breast cancer molecular subtyping and treatment progress. Breast Cancer Res. $2020 ; 22(1): 61$. doi:10.1186/s13058-020-01296-5

7. Bendewald MJ, Wetter DA, Li X, Davis MD. Incidence of dermatomyositis and clinically amyopathic dermatomyositis: a population-based study in Olmsted County, Minnesota. Arch Dermatol. 2010;146(1):26-30. doi:10.1001/archdermatol.2009.328

8. Sasaki H, Kohsaka H. Current diagnosis and treatment of polymyositis and dermatomyositis. Mod Rheumatol. 2018;28(6):913-921. doi:10.1080/ 14397595.2018 .1467257

9. Yang SH, Chang C, Lian ZX. Polymyositis and dermatomyositis - challenges in diagnosis and management. J Transl Autoimmun. $2019 ; 2: 100018$. doi:10.1016/j.jtauto.2019.100018

10. Piras M, Panebianco M, Garibaldi M, et al. A case of pathological complete response and resolution of dermatomyositis following neoadjuvant chemotherapy in HER2-positive early breast cancer. Curr Oncol. 2021;28(3):1957-1961. doi:10.3390/curroncol28030182

11. Chang L, Zhang L, Jia H, Nie Z, Zhang L. Malignancy in dermatomyositis: a retrospective paired case-control study of 202 patients from Central China. Medicine (Baltimore). 2020;99(34):e21733. doi:10.1097/MD.0000000000021733

12. Hendren E, Vinik O, Faragalla H, Haq R. Breast cancer and dermatomyositis: a case study and literature review. Curr Oncol. 2017;24(5):e429-e33. doi:10.3747/co.24.3696 
13. Cobos GA, Nelson CA, Alsarheed A, et al. Capecitabine-related eruption mimicking dermatomyositis in 2 patients with metastatic breast cancer. JAMA Dermatol. 2020;156(1):103-104. doi:10.1001/jamadermatol.2019.3162

14. Lu X, Yang H, Shu X, et al. Factors predicting malignancy in patients with polymyositis and dermatomyositis: a systematic review and meta-analysis. PLoS One. 2014;9(4):e94128. doi:10.1371/journal.pone.0094128

15. Hu T, Vinik O. Dermatomyositis and malignancy. Can Fam Physician. 2019;65(6):409-411.

16. Alenzi FM. Myositis specific autoantibodies: a clinical perspective. Open Access Rheumatol. 2020;12:9-14. doi:10.2147/OARRR.S231195

17. DeWane ME, Waldman R, Lu J. Dermatomyositis: clinical features and pathogenesis. J Am Acad Dermatol. 2020;82(2):267-281. doi:10.1016/j. jaad.2019.06.1309

18. Casciola-Rosen L, Nagaraju K, Plotz P, et al. Enhanced autoantigen expression in regenerating muscle cells in idiopathic inflammatory myopathy. $J$ Exp Med. 2005;201(4):591-601. doi:10.1084/jem.20041367

19. Fujimoto M, Watanabe R, Ishitsuka Y, Okiyama N. Recent advances in dermatomyositis-specific autoantibodies. Curr Opin Rheumatol. 2016;28 (6):636-644. doi:10.1097/BOR.0000000000000329

20. Zampieri S, Valente M, Adami N, et al. Polymyositis, dermatomyositis and malignancy: a further intriguing link. Autoimmun Rev. 2010;9 (6):449-453. doi:10.1016/j.autrev.2009.12.005

21. Primiano G, Plantone D, Sauchelli D, et al. Resolution of muscle inflammation after tumor removal in a woman with paraneoplastic dermatomyositis. $J$ Rheumatol. 2012;39(12):2359-2360. doi:10.3899/jrheum.120806

22. Racanelli V, Prete M, Minoia C, Favoino E, Perosa F. Rheumatic disorders as paraneoplastic syndromes. Autoimmun Rev. 2008;7(5):352-358. doi:10.1016/j.autrev.2008.02.001

23. Chaudhary LN, Wilkinson KH, Kong A. Triple-negative breast cancer: who should receive neoadjuvant chemotherapy? Surg Oncol Clin N Am. 2018;27(1):141-153. doi:10.1016/j.soc.2017.08.004

24. Bogdanov I, Kazandjieva J, Darlenski R, Tsankov N. Dermatomyositis: current concepts. Clin Dermatol. 2018;36(4):450-458. doi:10.1016/j. clindermatol.2018.04.003

25. Dias LP, Faria AL, Scandiuzzi MM, Inhaia CL, Shida JY, Gebrim LH. A rare case of severe myositis as paraneoplastic syndrome on breast cancer. World J Surg Oncol. 2015;13:134. doi:10.1186/s12957-015-0534-5

26. Sandhu NP, Zakaria S, Degnim AC, Boughey JC. Dermatomyositis presenting as a paraneoplastic syndrome due to underlying breast cancer. $B M J$ Case Rep. 2011;2011:bcr1020103416-bcr1020103416. doi:10.1136/bcr.10.2010.3416

27. Inaguma G, Shimada A, Tsunoda J, et al. Inflammatory breast cancer associated with amyopathic dermatomyositis: a case report. Surg Case Rep. 2020;6(1):284. doi:10.1186/s40792-020-01066-7

28. Pellegrino B, Mazzaschi G, Madeddu D, et al. Clinico-immunological profile of a 67-year-old woman affected by HER2-positive breast cancer and autoimmune dermatomyositis. Front Oncol. 2020;10:192. doi:10.3389/fonc.2020.00192

29. Zhang L, Zhang C, Yang Z, et al. Male occult triple-negative breast cancer with dermatomyositis: a case report and review of the literature. Onco Targets Ther. 2017;10:5459-5462. doi:10.2147/OTT.S151260

30. Chen AM, Obedian E, Haffty BG. Breast-conserving therapy in the setting of collagen vascular disease. Cancer J. 2001;7(6):480-491.

Cancer Management and Research

Dovepress

\section{Publish your work in this journal}

Cancer Management and Research is an international, peer-reviewed open access journal focusing on cancer research and the optimal use of preventative and integrated treatment interventions to achieve improved outcomes, enhanced survival and quality of life for the cancer patient. The manuscript management system is completely online and includes a very quick and fair peer-review system, which is all easy to use. Visit http://www.dovepress.com/testimonials.php to read real quotes from published authors.

Submit your manuscript here: https://www.dovepress.com/cancer-management-and-research-journal 